\title{
Aktuelles zur Klagebefugnis nach dem USchadG
}

\author{
Anmerkung zu dem Beschluss des VGH München vom 7.4.2015 - 8 CE 15.398, \\ NuR 2015, 336 (in diesem Heft)
}

\section{Erich Gassner}

(C) Springer-Verlag 2015

Der Antragsteller, ein nach $\$ 3$ Umwelt-Rechtsbehelfsgesetz (UmwRG) anerkannter Naturschutzverein, wollte mit einer einstweiligen Anordnung den Antragsgegner zur Anordnung von Sanierungsmaßnahmen nach dem Umweltschadensgesetz (USchadG) verpflichten. Der VGH München hat mit Beschluss vom 7. April 2015 den Antrag abgelehnt, weil das Umweltschadensgesetz in dem betreffenden Fall nicht anwendbar sei.

In der Sache geht es darum, den Abfluss von Berggrundwasser - als Folge eines Tunnelbaus - auf das Maß zu beschränken, das im Planfeststellungsbeschluss (PFB) vorgegeben ist.

Die Richter stellten - in dem zu entscheidenden Fall - in Abrede, dass eine berufliche Tätigkeit gemäß Anlage $1 \mathrm{zu}$ \3 Abs. 1 USchadG vorliege (a) und, dass der - unstreitig gegebene - Umweltschaden im Sinne des $\$ 19$ BNatSchG gemäß $₫ 3$ Abs. 1 Nr. 2 USchadG schuldhaft herbeigeführt worden sei (b).

Der Beschluss gibt Anlass, dazu kritisch Stellung zu nehmen.

a) Ein Umweltschaden im Sinne des Umweltschadengesetzes, im vorliegenden Fall geht es um ein Grundwasserschaden, vgl. \$90 Abs. 1 Nr. 3 WHG, liegt nur vor, wenn er durch eine berufliche Tätigkeit verursacht wird und diese Tätigkeit in Anlage $1 \mathrm{zu} \$ 3$ Abs. 1 USchadG gelistet ist. Letzteres ist in Bezug auf den hier erfolgten Straßenbau nicht der Fall. Indes ist der Grundwasserschaden nicht durch Spezifika des Straßenbaus, sondern durch das bedingt, was die gehobene wasserrechtliche Erlaubnis gestattet, die im Planfeststellungsbeschluss (PFB) aufgeführt wird. Für das anfallende Drainagewasser aus dem Tunnel und dem Erkundungsstollen wurde eine solche Erlaubnis nach $\$ 3$ Abs. 1 Nr. 5 WHG a.F. erteilt. Der PFB schreibt dezidiert vor, dass Wasserzutritte durch Injektionen auf das $\mathrm{Ma} \beta$ von $5 \mathrm{l} / \mathrm{sec}$ abzusenken sind. In diesem Zusammenhang ist staunenswert, dass der Beschluss - insoweit - eine Ermessensreduzierung auf Null ausschließt, weil es dem Vorhabensträger unbenommen bleibe, in einem Planänderungsverfahren andere als die im PFB vorgesehenen Maßnahmen zu ergreifen. Hier ist festzuhalten, dass der (nach wie vor in Kraft befindliche) PFB selbst von einer beruflichen Tätigkeit ausgeht, die in Nr. 5 der Anlage $1 \mathrm{zu} \$ 3$ Abs. 1 USchadG gelistet ist.

Dr. Erich Gassner, Ministerialrat a.D.,

Bonn, Deutschland
Die Richter verneinen allerdings ein zielgerichtetes Verhalten, da es sich um einen ungewollten Wasseraustritt handle. Dagegen ist einzuwenden, dass es darauf ankommt, was in der gehobenen Erlaubnis geregelt ist. Darin werden - antragsgemäß - zielgerichtete, d.h. an Sinn und Zweck des WHG ausgerichtete Maßnahmen genehmigt. Zusammen mit Maßnahme „M 2“ des PFB besteht somit eigens ein Regime, das präzise sagt, was - gezielt - zu tun ist, wenn bspw. die Grenze von 5 1/sec. überschritten wird. In Rede steht eine hochprofessionelle Tätigkeit, die wasserrechtlich eingeht und planungsrechtlich gesteuert wird.

Der Beschluss beruft sich nicht nur zu Unrecht auf Peters ${ }^{1}$, sondern setzt sich auch über $\$ 2 \mathrm{Nr}$. 4 USchadG hinweg. Dort wird ausdrücklich festgestellt, dass es nicht darauf ankommt, ob die Tätigkeit privat oder öffentlich, ob sie mit oder ohne Erwerbscharakter ausgeübt wird. Zudem ist Anlage $1 \mathrm{zu} \$ 3$ Abs. 1 USchadG zu entnehmen, dass es auf das Gefährdungsprofil der jeweiligen Tätigkeit ankommt. Die von den Richtern ins Feld geführten Kriterien der öffentlichen Aufgabe Daseinsvorsorge und der schlichten Hoheitsverwaltung finden im USchadG keine Stütze.

b) Umweltschäden im Sinne von $\$ 19$ BNatSchG setzen eine berufliche Tätigkeit voraus, diese braucht jedoch gemäß $\$ 3$ Abs. 1 Nr. 2 USchadG nicht gelistet zu sein. Insoweit ist entscheidend, ob der Schaden schuldhaft herbeigeführt worden ist. Dieses Kriterium wird vorliegend offensichtlich erfüllt. Der PFB normiert konkret-individuell die Schwelle von $5 \mathrm{l} / \mathrm{sec}$. Diese wird unstreitig massiv überschritten. Die darin zum Ausdruck kommende willentliche Hinnahme der Normverletzung wird trotz der beharrlichen Gegenvorstellungen der anerkannten Naturschutzvereinigung seitens des Freistaates Bayern ebenso beharrlich fortgesetzt. Dies zu brandmarken, ist ein wesentlicher Teil der Begründung des Antrags nach $\$ 123$ VwGO. ${ }^{2}$

Bemerkenswert ist, dass der Beschluss einerseits auf den sehr hohen finanziellen Aufwand für den Stopp der Grundwasserabsenkung hinweist, andererseits den Streitwert für das Beschwerdeverfahren auf 7500 Euro festsetzt.

Zusammenfassend ist zu bedauern, dass ein ökologisch so bedeutsamer Vorgang wie der hier besprochene - angesichts der ernsthaften Bemühungen eines anerkannten Sachwalters - prozessrechtlich so stiefmütterlich behandelt worden ist.

1) NuR 2014, 525, 529.

2) Vgl. im Übrigen Eyermann/ Happ, VwGO, 14. Aufl. (2014), \$123, Rdnr. 56. 